\title{
RHEUMATOID VASCULITIS - REPORT OF A CASE SATISFACTORIALLY TREATED WITH ANTI-TNF
}

\author{
Isabela Botelho Maciel Bueno ${ }^{1, *}$, Fernanda Miranda Caliani Sá1 ${ }^{1}$, Luiza Vasconcelos Schaefer ${ }^{1}$, Jéssica Pareja Guerra ${ }^{1}$ \\ 1. Hospital Regional de Presidente Prudente, Presidente Prudente (SP), Brazil. \\ ${ }^{\star}$ Corresponding author: isabelabmb@yahoo.com.br
}

\section{BACKGROUND}

Rheumatoid arthritis (RA) is a systemic inflammatory disease that has an estimated prevalence of 0.5 to $1 \%$ of the world population, with a higher incidence in the age group of 30 to 50 years, preferably in women. In addition to the classic clinical presentation with joint involvement, the disease can develop with extraarticular involvement, as in the case with rheumatoid vasculitis. The choice of therapy is based on the severity and intensity of the manifestations.

\section{CASE REPORT}

Patient D.L.F.J., 51 years old, female, with previous diagnosis of rheumatoid arthritis, being followed up with a rheumatologist, using leflunomide 20 mg for 5 years. She was admited in the hospital on January 22019 complaining of paresthesia in the lower limbs that evolved to cyanosis of the extremities. There was worsening of the cyanosis and the appearance of hyperemic lesions in the left lower limb that have evolved into ulcerated and necrotic lesions in the same limb (Fig. 1). The biopsy of the skin lesion showed a thin epidermis, with rectification of interpapillary ridges, the hypodermis presented a slight thickening of the fibrous septa, permeated by an inflammatory infiltrate made up of neutrophils, being conducted as small vessel vasculitis. Initially, treatment with azathioprine, corticotherapy and topical medications was performed. The patient presented little improvement in the lesions (Fig. 2) and in the condition of arthralgia, being opted for the start of immunobiological (anti-TNF). Seven days after the first application, the lesions improved, with significant improvement after the third dose (Fig. 3), in addition to an improvement in the joint condition, including returning to walk. A gradual reduction in the dose of corticosteroids was initiated. The patient remained well, with complete healing of the lesions and with significant improvement in arthralgia and walking.

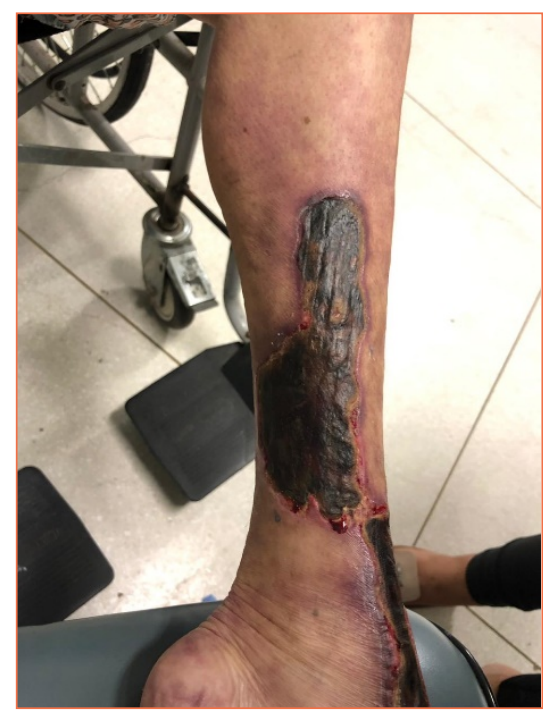

Figure 1. Necrotic plaques in March 2019. 


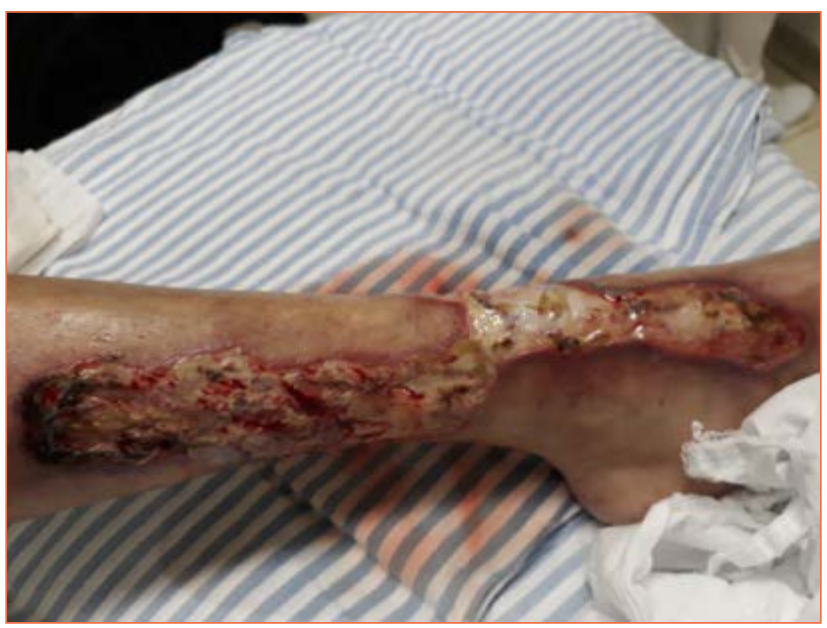

Figure 2. Lesion with granulation tissue in April 2019.

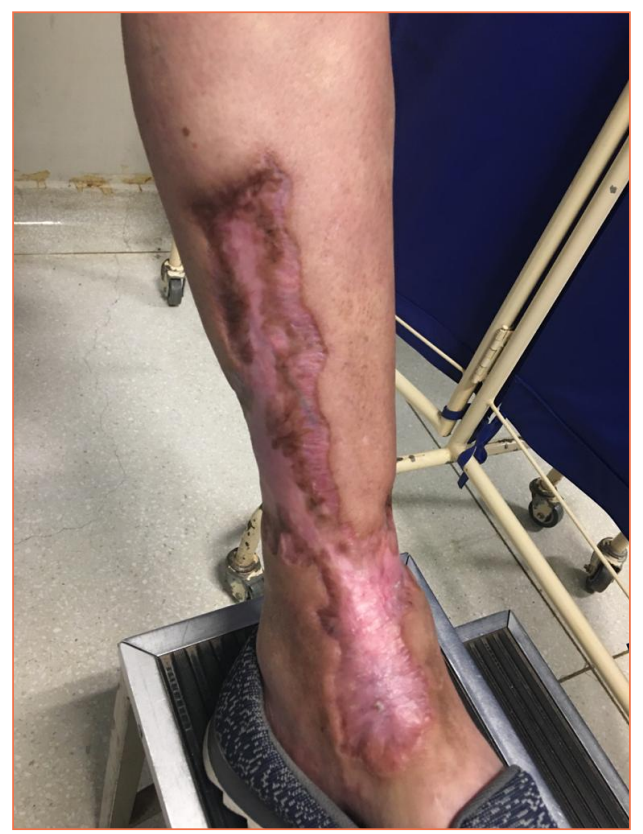

Figure 3. Lesions in an advanced healing process after using anti-TNF in July 2019.

\section{CONCLUSION}

This case showed us a RA patient with rheumatoid vasculitis with severe skin involvement refractory to synthetic DMARD and with a quick and sustained response with anti-TNF, demonstrating its effectiveness in this condition.

*The patient authorized the disclosure of the information and images contained in the report by signing the informed consent form. 